ESJ Social Sciences

\title{
Pratiques Liées aux Représentations Sociales des Excréments Humains chez les Populations des Quartiers Défavorisés du District d'Abidjan en Côte d'Ivoire
}

\author{
BLA Bénédicte Sonia, Doctorante en sociologie de l'environnement
}

Institut d'Ethnosociologie (I.E.S), Université Félix HOUPHOUËT-

BOIGNY, Abidjan (Côte d'Ivoire), Laboratoire d'Étude et de Recherche Interdisciplinaires en Sciences Sociales (LERISS)

Doi:10.19044/esj.2021.v17n32p166

Submitted: 09 June 2021

Accepted: 02 September 2021

Published: 30 September 2021
Copyright 2021 Author(s)

Under Creative Commons BY-NC-ND

4.0 OPEN ACCESS

Cite As:

BLA Bénédicte S. (2021). Pratiques Liées aux Représentations Sociales des Excréments Humains chez les Populations des Quartiers Défavorisés du District d'Abidjan en Côte d'Ivoire. European Scientific Journal, ESJ, 17 (32), 166.

https://doi.org/10.19044/esj.2021.v17n32p166

\section{Résumé}

La gestion des excréments humains révèle des pratiques à risque pour l'environnement en général et pour la santé des populations en particulier. D'où l'intérêt de cette étude dans les quartiers défavorisés où les difficultés en assainissement sont plus accentuées. La présente recherche s'inscrit dans le cadre d'une approche mixte. Pour ce faire, les méthodes et les techniques correspondant à l'approche qualitative et quantitative ont été utilisées pour collecter les données. Il s'agit, de la grille d'observation, du guide d'entretien et du questionnaire. L'objectif est d'analyser les représentations sociales des excréments humains dans les quartiers défavorisés du District d'Abidjan précisément à bougounisso, sagbé antenne, gbebouto, grand campement, tofiato et zimbabwé. La théorie des représentations sociales développée par Bourdieu a été mobilisée à cet effet. Elle a permis de mettre en exergue les représentations sociales des excréments humains chez les populations des quartiers défavorisés. Les résultats ont montré que la gestion des excréments humains est animée par un ensemble d'idéologie qui se résume en des croyances, des tabous et des sanctions. Il ressort que les différentes définitions des excréments humains se rapportent à la saleté, à l'impureté, aux déchets. Ils sont associés à tout ce qui est synonyme de mal, maladie, malédiction, malheur. Les croyances en rapport avec la gestion des excréments humains 
sont observées sur le plan religieux et culturel. Ce sont des dispositions relatives au choix du lieu de défécation et des règles d'hygiènes que le croyant doit observer. Les tabous concernent les lieux publics où il est défendu de déféquer.

Mots-clés: Représentations sociales, gestion des excréments humains, environnement, quartiers défavorisés, District d'Abidjan

\title{
Practices Related to Social Representations of Human Excreta in Disadvantaged Neighborhoods of the District of Abidjan, Ivory Coast
}

\section{BLA Bénédicte Sonia, Doctorante en sociologie de l'environnement} Institut d'Ethnosociologie (I.E.S), Université Félix HOUPHOUËTBOIGNY, Abidjan (Côte d'Ivoire), Laboratoire d'Étude et de Recherche Interdisciplinaires en Sciences Sociales (LERISS)

\begin{abstract}
The management of human excreta reveals risky practices for the environment in general and for the health of populations in particular. Hence the interest of this study in disadvantaged neighborhoods where sanitation difficulties are more accentuated. This research subscribe to a mixed approach. To be done, the methods and techniques corresponding to the qualitative and quantitative approach were used to collect the data. These are observation grid, the interview guide and the questionnaire. The objective is to analyse the social representations of human excreta in disadvantaged neighborhoods of the District of Abidjan specifically in bougounisso, sagbé antenne, gbebouto, grand campement, tofiato and zimbabwé. The theory of social representations developed by Bourdieu was used for this purpose. It has made it possible to highlight the social representations of human excreta among populations in disadvantaged neighborhoods. The results showed that in traditional societies the management of human faeces is driven by a set of ideologies that boil down to beliefs, taboos and sanctions developed around human faeces and the act of defecation. It appears that the different definition of human excreta relate to dirt, impurity and waste. They are associated with everything that is synonymous with evil; disease, curse, misfortune. Beliefs related to the management of human excreta are observed on a religious and cultural level. These provisions relating of the choice of the place of defecation and the rules of hygiene that the believer must observe. The taboos refers to public places where defecation is forbidden.
\end{abstract}


Keywords: Social representations, management of human excreta, environment, disadvantaged neighborhoods, District of Abidjan

\section{Introduction}

Excréments et urines sont une production naturelle importante (N'Donko, 1988). Ils désignent l'ensemble des matières fécales évacuées par le corps de l'homme après consommation, une autre appellation est celle de excréta (Le Grand Larousse, 2017). Aborder la question des excréments n'est pas chose facile. En effet, la défécation est devenue sujet de honte impliquant le silence, le repli sur soi et surtout la crainte d'être surpris en si fâcheuse posture ( Norbert Elias, 1969 cité par Berge et al, 2010). Du fait de leurs caractères répugnants, parler des excréments humains ou de la défécation nécessite de prendre certaines dispositions. En effet, les excréments et particulièrement les fèces humaines sont chargées de fortes connotations négatives dues à leur répugnance visuelle et olfactive et également aux maladies et malheurs qu'ils sont susceptibles de causer (N'Goran, 2016). La nature même des excréments d'une part, les représentations sociales qui les accompagnent d'autre part ont alimenté le sentiment de rejet vis-à-vis de ces déchets. De nombreux auteurs ont travaillé sur la question. Au nombre de ceux-ci, figurent N'Goran (2016), Berger et al (2010) ; Le Breton (1997) et N'Donko (1988) qui ont abordé les représentations sociales des excréta. D'autres comme Elias (1976) ; Bourke (1981) et Lawer (2002) ont évoqué l'histoire de la gestion des excréments humains.

Les sociétés disposent d'une ligne de conduite qui organise la gestion des excréments humains. La défécation est une activité physiologique commune à tous les hommes, quel que soit leur condition, leur culture. Cet acte banal qui se veut très intime s'inscrit pourtant dans toute une réglementation sociale (N'Donko, 1988). Cependant, plus de 200 millions de tonnes de déchets humains sont déversés dans les cours d'eau chaque année au niveau mondial, réduisant tant quantitativement que qualitativement l'eau nécessaire à la consommation (UNICEF, 2008).

En Côte d'Ivoire, $28 \%$ de la population totale, soit 6,33 millions de personnes, pratiquent la défécation à l'air libre (INS, 2016; Ministère du Plan et du Développement [MPD], 2016). Dans le district d'Abidjan la situation est encore plus grave dans les quartiers précaires. En effet, selon le PNUD, $30 \%$ des populations de ces quartiers défèquent à l'air libre. Les lieux indiqués sont les broussailles, les bordures de la lagune ou de la mer mettant en lumière une absence de gestion des excréta (PNUD, 2013).

Pourtant, dans les quartiers où a été mené cette recherche, des projets ont été entrepris aussi bien par les ministères que les Organismes Non Gouvernementaux (ONG) dans le sens d'une gestion efficiente des excréta 
afin d'améliorer le cadre de vie des populations. À cet effet, dans la commune d'Abobo les populations de sagbé antenne ont bénéficié de la part de l'UNCEF de construction de latrine. Dans cette même veine, la mairie de Port-Bouët a construit dans les quartiers défavorisés des WC publics. À bougounisso, toujours dans la commune d'Abobo, le Centre de Recherche pour la Paix (CERAP) en appui avec la mairie d'Abobo et l'ONU habitat ont mené des activités sur l'assainissement avec à l'appui une remise de matériel.

Par ailleurs, pour améliorer les conditions environnementales et sanitaires de ces populations, l'Office National d'Assainissement et de Drainage (ONAD) en accord avec le ministère de tutelle mènent des opérations de curage et d'entretien des caniveaux ainsi que d'autres ouvrages d'assainissement, des campagnes de sensibilisation aux bonnes pratiques d'hygiènes à travers les médias, les affiches dans les rues, l'actualisation du schéma directeur de l'assainissement. Malgré toutes ces actions posées en vue d'arriver à une gestion efficiente des déchets humains, des pratiques à risque ont été identifiées dans ces quartiers. Ce sont : le déversement du contenu des fosses ou puits perdus issu de la vidange des latrines à proximité des lieux d'habitations, le débordement des fosses septiques, la défécation à l'air libre.

De ce fait, il convient de s'intéresser aux représentations sociales des excréments humains chez les populations des quartiers défavorisés. La représentation désigne, en priorité une vaste classe de formes mentales (sciences, religions, mythes, espaces, temps), d'opinions et de savoirs sans distinction. (...). Elle a une certaine fixité, s'agissant d'un concept, et une objectivité, puisqu'elle est partagée et reproduite de manière collective. Voilà qui lui donne le pouvoir de pénétrer dans chaque individu, comme du dehors, et de s'imposer (Moscovici, 1989 :65, cité N'Guessan, 2016). C'est un ensemble de connaissances, d'attitudes, de croyances concernant un objet donné. Elle comprend des savoirs, des prises de position, des applications de valeurs, des prescriptions normatives (Flament et Rouquette, 2003, p. 13). Elle oriente et organise les conduites et les communications sociales au sein d'une communauté. Les individus sont guidés dans leur agir par les représentations qu'ils ont d'un objet ou d'un fait social présent dans leur environnement (Garnier et Sauvé, 1999 ; Jodelet, 1989 cités par Kouadio et al, 2021).

Partant, il est important de se poser la question suivante : quelles sont les représentations sociales des excréments humains chez les populations de bougounisso, sagbé antenne, gbebouto, grand campement, tofiato et zimbabwé ? Pour répondre à cette question, l'hypothèse suivante a été émise : La gestion des excréments humains est liée aux croyances et tabous des populations. Cette recherche vise à identifier les représentations sociales des excréments humains chez les populations des quartiers de bougounisso, sagbé antenne, gbebouto, grand campement, tofiato et zimbabwé. La théorie des représentations sociales a été mobilisé à cet effet. 


\section{Méthodologie}

Il s'agit de présenter la démarche scientifique qui a accompagné la réalisation de cette recherche. Elle comporte la délimitation du champ et de la population à l'étude, l'échantillon, les outils et techniques de collecte de données, le traitement et analyse des données.

\subsection{Présentation du cadre de la recherche}

Cette recherche a été réalisée dans six quartiers de quatre des treize communes qui composent le District d'Abidjan à savoir : bougounisso et sagbé antenne pour la commune d'Abobo, gbebouto à Attécoubé, grand campement à Koumassi, tofiato et zimbabwé dans la commune de Port-Bouët. Le District Autonome d'Abidjan (DAA) est situé dans le sud de la Côte d'Ivoire, au bord du Golfe de Guinée. Il est limité par les communes de Jacqueville, Grand-Lahou et Dabou à l'Ouest, les communes de Sikensi, Tiassalé, Agboville, Adzopé et Alépé au Nord, la commune de Grand-Bassam à l'Est et au Sud par l'Océan Atlantique. Le District Autonome d'Abidjan comprend les dix communes qui composent la ville d'Abidjan, à savoir : Abobo, Adjamé, Attécoubé, Cocody, Koumassi, Marcory, Plateau, PortBouët, Treichville, Yopougon, auxquelles s'ajoutent les trois sous-préfectures que sont Anyama, Bingerville et Songon.

Le choix du District d'Abidjan s'est fait en raison du fait qu'il concentre un nombre important de quartiers défavorisés. Les dernières études en ont dénombré cent trente-sept (137) (PNUD, 2013). Le choix des quartiers se justifie d'une part par le faible niveau d'équipement d'assainissement, d'autre part par le fait que les pratiques à risques de la gestion des excréments humains soient plus accentuées. 
Carte $\mathbf{N}^{\circ} 1$ : Présentation de la zone d'étude

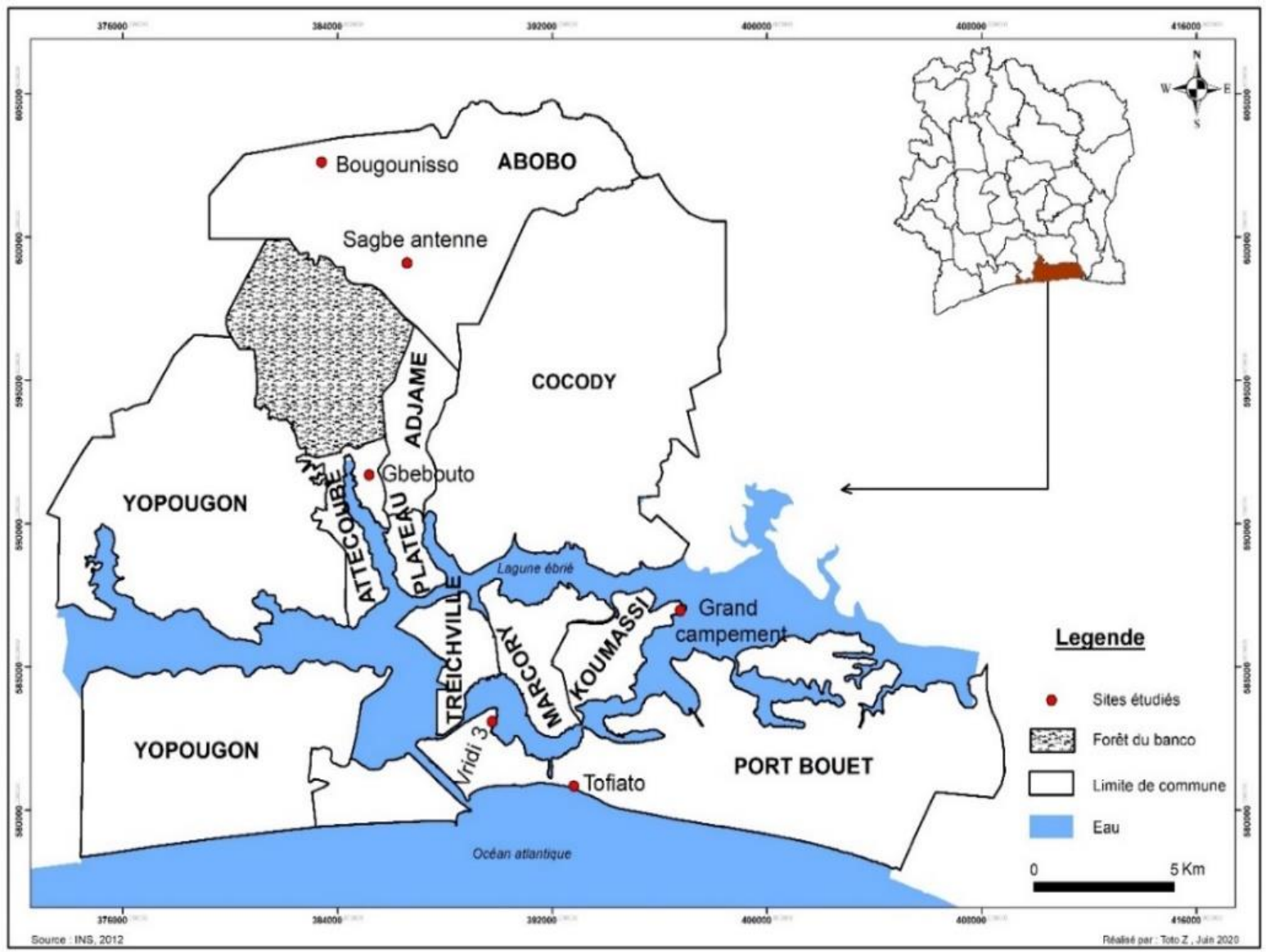

Source : Institut National de la Statistique, 2012

\subsection{Population à l'étude}

La première catégorie d'enquêtés est la population des six quartiers précaires soumis à l'étude. Les populations constituent les acteurs clés de la gestion des excréta. La deuxième catégorie concerne les responsables des structures institutionnelles de l'assainissement tels que le ministère en charge de l'assainissement, la direction assainissement du District d'Abidjan, le service technique de la mairie d'Abobo, d'Attécoubé, de Koumassi et de PortBouët. La troisième catégorie est composée des opérateurs économiques qui interviennent dans le secteur de l'assainissement des eaux usées domestiques telles que le service de vidange, les gérants de toilettes publiques.

\subsection{Composition de l'échantillon}

L'approche étant mixte, l'échantillon est à la fois qualitatif et quantitatif. L'échantillonnage par choix raisonné a été choisi dans le cadre de 
l'échantillonnage qualitatif. Le critère de choix est essentiellement la profession des enquêtés. Il implique d'interroger des professionnels du secteur de l'assainissement des excréta et/ou des eaux usées. Ainsi, les cibles étaient les services de vidange, les gérants des toilettes publiques. Pour ce qui est des acteurs institutionnels, le Ministère de la construction, du Logement, de l'Assainissement et de l'Urbanisme (MCLAU), les services techniques des mairies de Port-Bouët, Koumassi, Attécoubé et Abobo. À l'issu des entretiens, les cibles interrogées sont : un service de vidange, quatre gérants de toilette publique, un agent de la direction assainissement, un agent de la direction urbanisme, un agent de la direction logement du MCLAU, un responsable dans chaque service technique des différentes mairies. Aussi, des focus group avec les populations de zimbabwé, tofiato, sagbé antenne, gbebouto ont complétés les entretiens individuels. Ces populations ont été représentés par les chefs communautaires. Le tableau qui suit résume le nombre et le type d'entretien réalisé par acteur.

Tableau $\mathbf{N}^{\circ} \mathbf{I}$ : Échantillonnage qualitatif

\begin{tabular}{|l|c|}
\hline \multicolumn{1}{|c|}{ CIBLES } & ENTRETIENS RÉALISÉS \\
\hline MCLAU & $\begin{array}{c}3 \text { entretiens individuels } \\
\text { - directeur direction assainissement du MCLAU } \\
\text { - chef service direction de l'urbanisme du MCLAU } \\
\text { - chef service direction logement du MCLAU }\end{array}$ \\
\hline $\begin{array}{l}\text { Service technique de la } \\
\text { mairie }\end{array}$ & $\begin{array}{c}\text { 4 entretiens individuels } \\
\text { - chef service hygiène et salubrité (mairie de } \\
\text { Koumassi) }\end{array}$ \\
$\begin{array}{l}\text { - Responsable service hygiène (mairie d'Attécoubé) } \\
\text { - Sous-directeur de l'environnement et cadre de vie } \\
\text { (mairie d'Abobo) }\end{array}$ \\
\hline Service de vidange \\
Gérant de toilettes publiques \\
$\begin{array}{l}\text { Chefs communautaires des } \\
\text { quartiers }\end{array}$
\end{tabular}

Source : Données de la recherche, juillet 2019

L'échantillon défini dans l'approche quantitative se veut être représentatif, pour se faire, la technique d'échantillonnage aléatoire simple a été retenue. La base de données sur les populations des quartiers choisis a été constituée à partir de l'extrapolation des données démographiques du 
recensement 1998. Ainsi la taille de l'échantillon total à enquêter dans cette étude est de 659.

Tableau $\mathbf{N}^{\circ}$ II : Échantillonnage quantitatif

\begin{tabular}{|c|c|c|c|}
\hline Communes & Quartiers défavorisés & \multicolumn{2}{|c|}{ Échantillon } \\
\hline \multirow{2}{*}{ Port-Bouët } & Tofiato & 32 & \multirow{2}{*}{91} \\
\cline { 2 - 3 } & Zimbabwé & 59 & \multirow{2}{*}{72} \\
\hline \multirow{2}{*}{ Abobo } & Sagbé Antenne & 24 & 126 \\
\hline Attécoubé & Bougounisso & 48 & 370 \\
\hline Koumassi & Gbebouto & 126 & 659 \\
\hline Total & Grand campement & 370 & 659 \\
\hline
\end{tabular}

Source : INS, données mises à jour en 2019, d'après le recensement de 1998

\subsection{Outils et techniques de collecte des données}

Les données de l'enquête qualitative ont été collectées à partir de la grille d'observation et du guide d'entretien. Ils portaient essentiellement sur : les pratiques de gestion et les représentations sociales des excréments humains. Quant aux données quantitatives, elles ont été collectées à partir du questionnaire. Les points abordés sont: les représentations sociales et les définitions des excréments humains. Au regard de ces outils, les techniques mobilisées sont la recherche documentaire, l'observation, l'entretien et le sondage.

\subsection{Traitement et Analyse des données}

Le traitement des données quantitatives a nécessité, l'élaboration d'un masque de saisie à l'aide du logiciel sphinx plus $^{2}$ (V5) grâce auquel l'enregistrement des données issues du questionnaire physique a été possible. Pour ce qui est de l'analyse, l'analyse par tableaux croisés a permis de déceler les relations entre les différentes variables. Les variables utilisées sont le sexe, la religion, le niveau d'instruction.

L'analyse de contenu thématique est la méthode choisie dans le cadre du traitement des données qualitatives. Celle-ci a été précédée par la retranscription des entrevues réalisées en vue de faire ressortir les verbatims en rapports avec les thématiques abordées. Les dimensions des représentations sociales des excréments humains sont symboliques et sociales. La première se traduit par l'existence de référents culturels faisant des fèces le symbole de la saleté et l'existence de système de valeur leur conférant des aspects maléfiques et les considérant comme chargées de connotations négatives. La seconde, se traduit par l'existence dans les sociétés de croyances sur les excréments humains, de pratiques sociales communes dans la gestion des fèces. De plus, ils sont socialement identifiés comme source de maladies et de malédictions. 
Les groupes sociolingustiques qui ont participés aux entretiens sont ceux organisés en communauté et représenté par un chef communautaire lors des focus group. Ainsi, les réponses des enquêtés ont été analysées en fonction des langues locales rencontrées.

\section{Résultats}

Les représentations sociales liées aux excréments humains mettent en exergue le jugement des populations vis-à-vis de leurs propres déjections. Il s'agit en claire, de saisir les définitions, les représentations, les croyances et les tabous qui accompagnent les selles.

\section{1 Définitions et représentations des excréments humains}

\subsubsection{Différentes définitions des excréments humains}

Le tableau $\mathrm{N}^{\circ}$ III présente les différentes définitions des excréments humains. Les variables qui définissent les excréments sont l'impureté, la saleté, les déchets.

Tableau $\mathbf{N}^{\circ}$ III : Répartition des enquêtés selon leur définition des excréments humains

\begin{tabular}{l|c|c|}
\hline & & \\
\hline $\begin{array}{l}\text { Définition des } \\
\text { excréments humains }\end{array}$ & Effectifs & Fréquence en \% \\
\hline Impureté & 175 & 26,6 \\
\hline Saleté & 268 & 40,7 \\
\hline Déchets & 211 & 32 \\
\hline Autre & 5 & 0,7 \\
\hline Total & 659 & 100 \\
\hline
\end{tabular}

Source : Données de la recherche, juillet 2019

Ce tableau résume le sens que les populations attribuent aux excréments humains. Ainsi, pour $40,7 \%$ des enquêtés, les excréments humains sont synonymes de saleté, $32 \%$ d'entre eux affirment que ce sont des déchets, par contre, 26,6 soutiennent que ce sont des impuretés. Il importe de mentionner que ces diverses définitions se rapportent à ce qui est malpropre ou à quelque chose qui souille une fois qu'on rencontre en contact avec. En effet, la saleté désigne la souillure du corps alors que l'impureté va au-delà et touche le spirituel. Ainsi, les résultats confirment le constat selon lequel les populations ont un jugement péjoratif des fèces. À ce sujet, un chef de communauté déclare: "Je pense à ce qui n'est pas bon que je vais dégager, ce que je ne peux pas conserver dans le ventre, c'est ce que je fais sortir. Je ne peux pas penser à quelque chose qui est bon. Je pense déjà à de la saleté qui va 
ressortir, c'est de la saleté. L'objectif c'est dégager la saleté du ventre pour être libéré. » Dans cette même veine, un autre participant affirme en ces termes: "Bô c'est la saleté, c'est un truc que je n'aime pas, que je vais dégager. Voilà pourquoi on dit bô. Bô en dioula, bambara signifie que je vais enlever quelque chose en moi pour aller jeter. On veut l'enlever pour aller jeter parce que ça gêne. »

De ces propos, il ressort que l'évocation du mot «bô » ou « caca » renvoie systématiquement à "tout ce qui est négatif et qui se rejette ». Les selles sont des choses avec lesquels l'homme ne peut pas vivre. De ce fait, il faut absolument s'en débarrasser. La nature répugnante des excréments, suscite le dégout une fois que le mot est prononcé.

\subsubsection{Définitions des excréments humains en fonction du sexe}

Le graphique ci-dessous met en relation les différentes définitions des excréments humains et le sexe des enquêtés.

Graphique $\mathbf{N}^{\circ} \mathbf{1}$ : Répartition de la définition des excréments humains selon le sexe des enquêtés

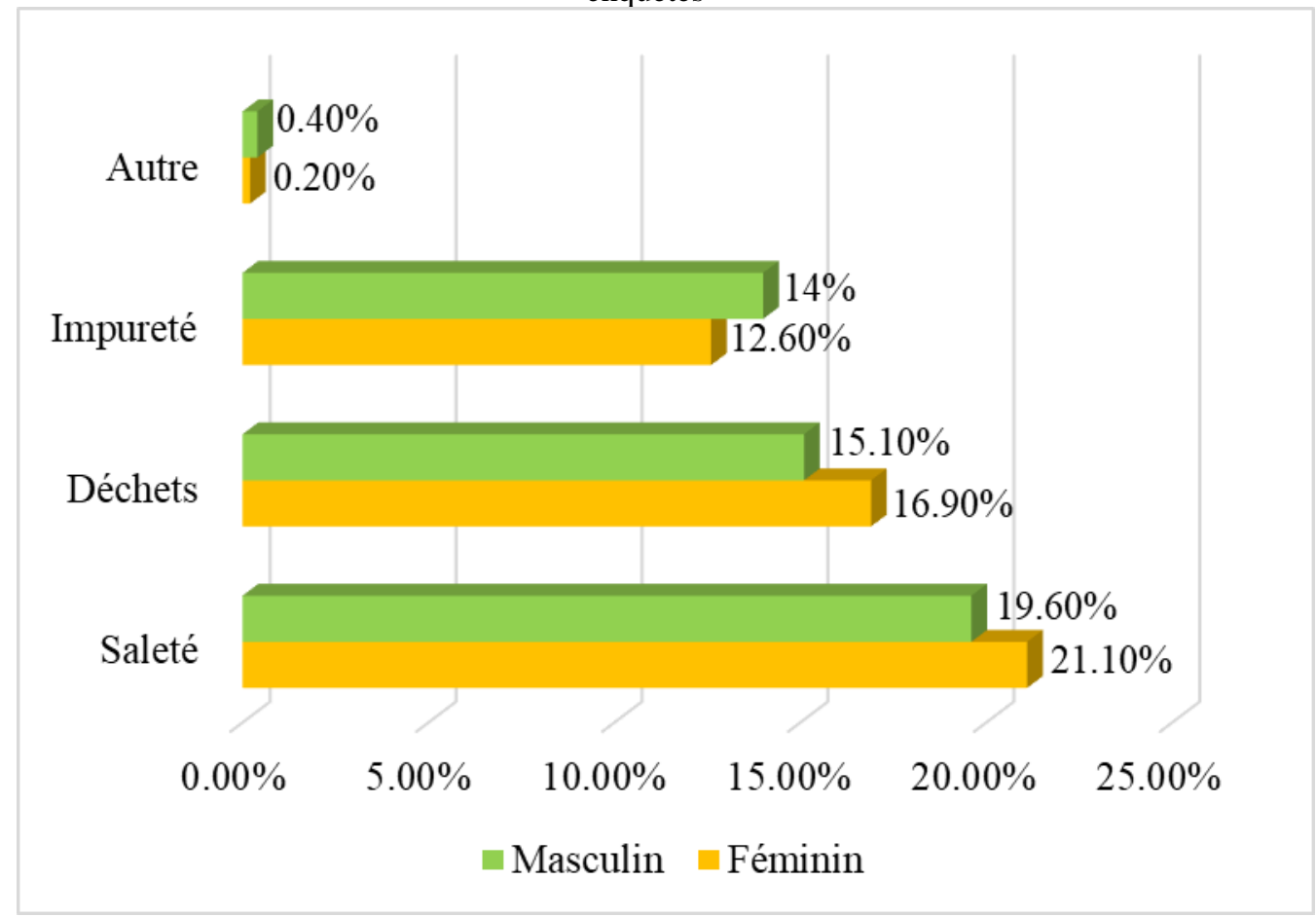

Source : Données de la recherche, juillet 2019

Ce graphique révèle qu'indifféremment du sexe, les personnes interrogées définissent à plus de $40 \%$ les excréments humains comme étant de la saleté, avec $21,1 \%$ de femme et $19,6 \%$ d'hommes. Il faut également souligner que $32 \%$ dont $16,9 \%$ de femmes définissent les excréments humains 
comme des déchets. Par ailleurs, pour $26,6 \%$ des enquêtés, les excréments humains se définissent par l'impureté. Cette tendance est dominée à $14 \%$ par les hommes.

En somme, il faut retenir que, en se fondant sur le sexe des enquêtés, les définitions des excréments humains sont sensiblement proches. Cependant, il faut noter deux tendances, l'une à domination masculine $(14 \% \mathrm{~d}$ 'homme définissent les excréments humains comme étant signe d'impureté contre $12,60 \%$ de femme) et l'autre à domination féminine $(38 \%$ de femme définissent les excréments humains comme des déchets ou de la saleté contre $34,7 \%$ d'homme).

\subsubsection{Définitions des excréments humains en fonction de la religion}

La répartition des enquêtés en fonction de leur définition des excréments humains et de leur religion est présentée dans le graphique suivant.

Graphique $\mathbf{N}^{\circ} \mathbf{2}$ : Répartition des enquêtés en fonction de leur définition des excréments humains et de leur religion

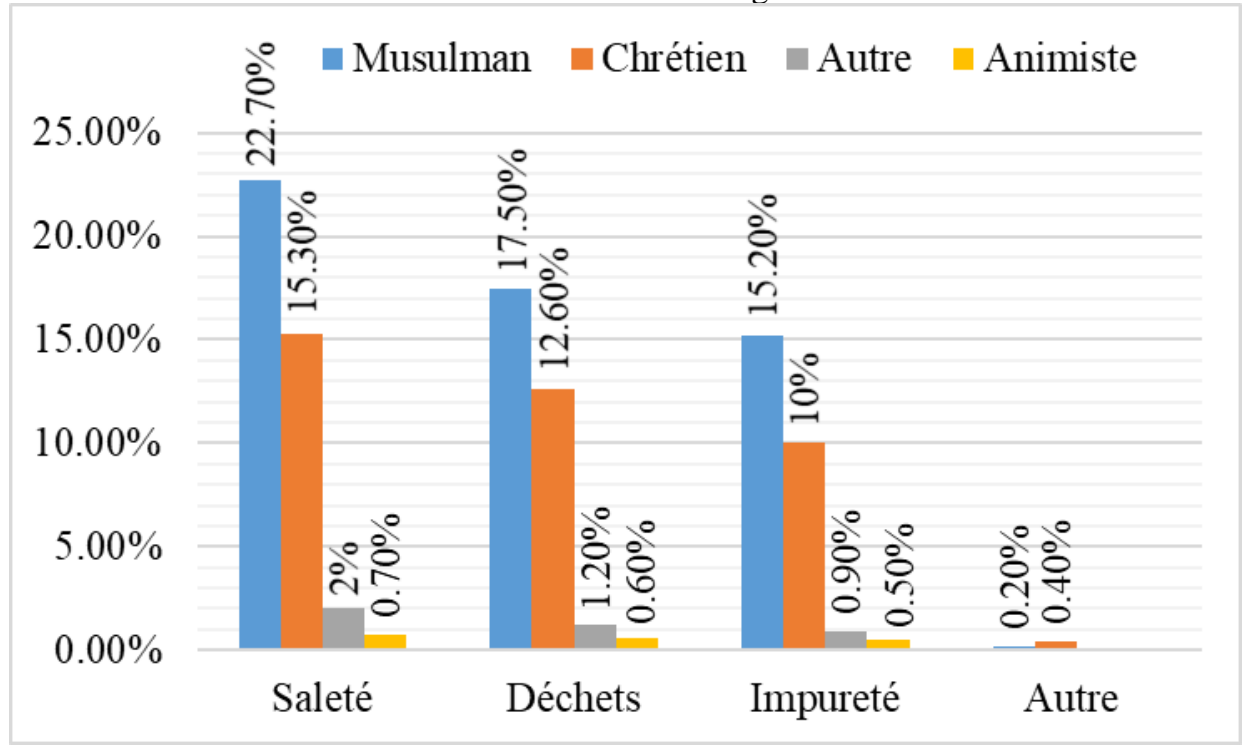

Source : Données de la recherche, juillet 2019

À travers ce graphique, il faut noter que la définition des excréments humains par les personnes interrogées, n'est pas influencée par la religion. En effet, les différents aspects de la définition des excréments humains sont cités par les personnes interrogées indifféremment de leur appartenance religieuse. Ainsi, 22,7\% des musulmans, 15,3\% des chrétiens, $2 \%$ des autres et $0,7 \%$ des animistes définissent les excréments humains comme étant de la saleté. Pour les enquêtés ayant défini les excréments humains comme des déchets ou l'impureté, la même tendance a été observée dans les réponses. Les autres concernent la catégorie de personnes qui ne s'identifient à aucune religion. 


\subsubsection{Définition des excréments humains en fonction du niveau d'instruction}

Le tableau $\mathrm{N}^{\circ} \mathrm{IV}$ traduit clairement le rapport définition des excréments humains/niveau d'instruction.

Tableau $\mathbf{N}^{\circ} \mathbf{I V}$ : Répartition des enquêtés en fonction de la définition des excréments humains et du niveau d'instruction

\begin{tabular}{|l|c|c|c|c|c|}
\hline \multicolumn{1}{|r|}{$\begin{array}{r}\text { Définition des excréments } \\
\text { humains }\end{array}$} & Impureté & Saleté & Déchets & Autre & TOTAL \\
$\begin{array}{l}\text { Niveau d'instruction } \\
\text { du répondant }\end{array}$ & $13,1 \%$ & $18,9 \%$ & $15,2 \%$ & $0,3 \%$ & $47,5 \%$ \\
\hline Aucun niveau & $6 \%$ & $10,4 \%$ & $7,6 \%$ & $0,2 \%$ & $24,2 \%$ \\
\hline Primaire & $4,5 \%$ & $7,7 \%$ & $5 \%$ & $0,1 \%$ & $17,3 \%$ \\
\hline Secondaire & $3 \%$ & $3,7 \%$ & $4,2 \%$ & 0 & $11 \%$ \\
\hline Supérieur & $26,6 \%$ & $40,7 \%$ & $32 \%$ & $0,6 \%$ & $100 \%$ \\
\hline TOTAL & & & & \\
\hline
\end{tabular}

Source : Données de la recherche, juillet 2019

En considérant le niveau d'instruction en rapport avec la définition des excréments humains, nous relevons à travers le tableau que les enquêtés quel que soit leur niveau d'instruction mentionnent plus la saleté, l'impureté et les déchets comme définition des excréta. La distribution des réponses montre que, quel que soit le niveau d'instruction, les excréments humains sont principalement définis à travers la « saleté » qui domine chez les personnes qui n'ont aucun niveau d'étude $(18,9 \%)$, de niveau primaire $(10,4 \%)$ et secondaire $(7,7 \%)$. Le second élément de réponse en termes de pourcentage est : "les déchets ». Cette tendance domine chez les personnes ayant atteint le niveau supérieur $(4,2 \%)$. Il convient de relever qu'à travers les réponses des enquêtés, quel que soit le niveau d'instruction, ils se réfèrent plus à ce qui est en rapport avec le physique comme la saleté et les déchets. 


\subsection{Excréments humains perçus comme synonyme de mal}

Graphique $\mathbf{N}^{\circ} \mathbf{3}$ : Répartition des enquêtés selon leurs représentations des excréments humains

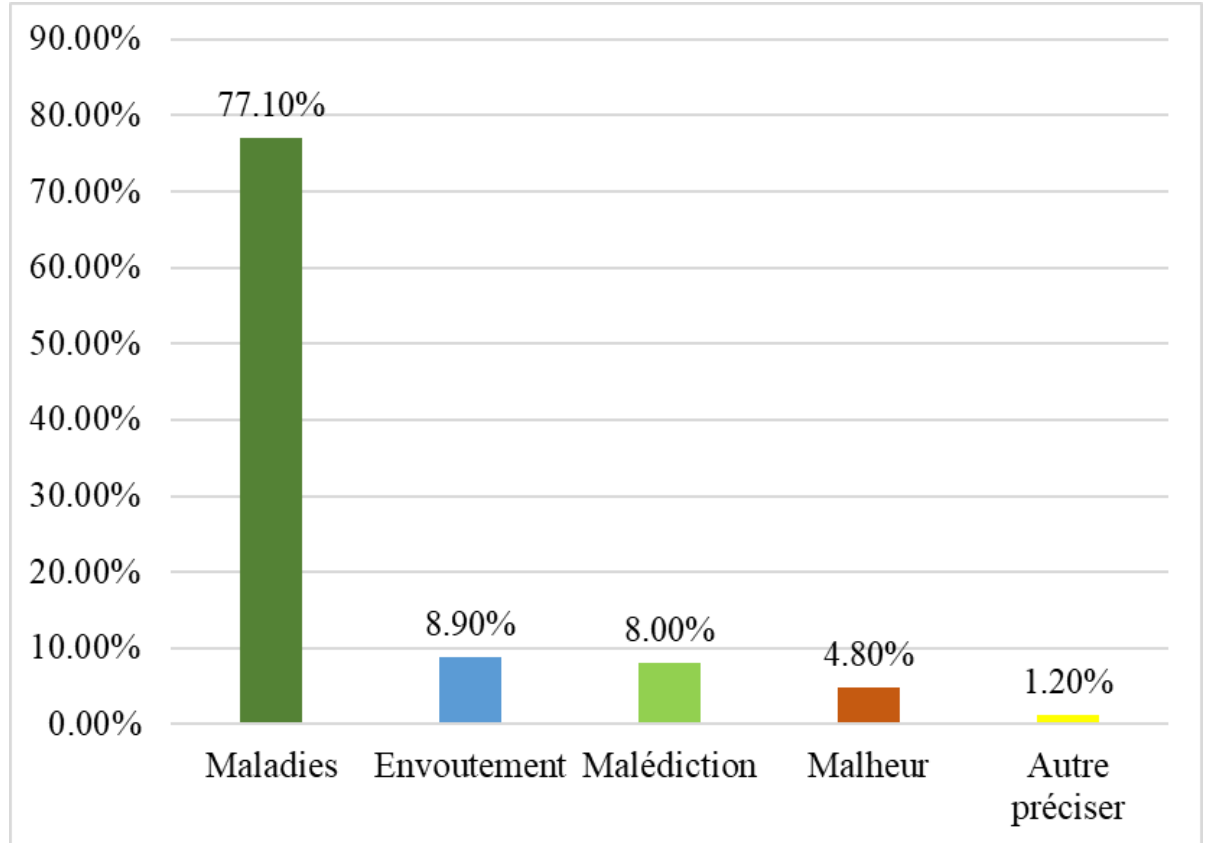

Source : Données de la recherche, juillet 2019

Chez les populations soumises à la recherche, les excréments humains sont associés à tout ce qui est nuisible comme la maladie, la malédiction, le malheur, l'envoutement. Au vu du graphique, il faut noter que pour $77,1 \%$ des enquêtés, les excréments sont synonymes de maladie. Par ailleurs, certaines personnes les associent à la malédiction ( $8 \%)$, à l'envoutement $(8,9 \%)$ et au malheur $(4,8)$.

Dans la conception africaine, les excréments humains ont une dimension mystique. Ainsi, les selles d'une personne peuvent être utilisées pour lui faire du mal. Cet aspect de notre travail sera développé un peu plus loin. 


\subsubsection{Représentations des excréments humains en fonction du sexe}

Graphique $\mathbf{N}^{\circ} \mathbf{4}$ : Répartition des enquêtés en fonction des représentations des excréments humains et du sexe

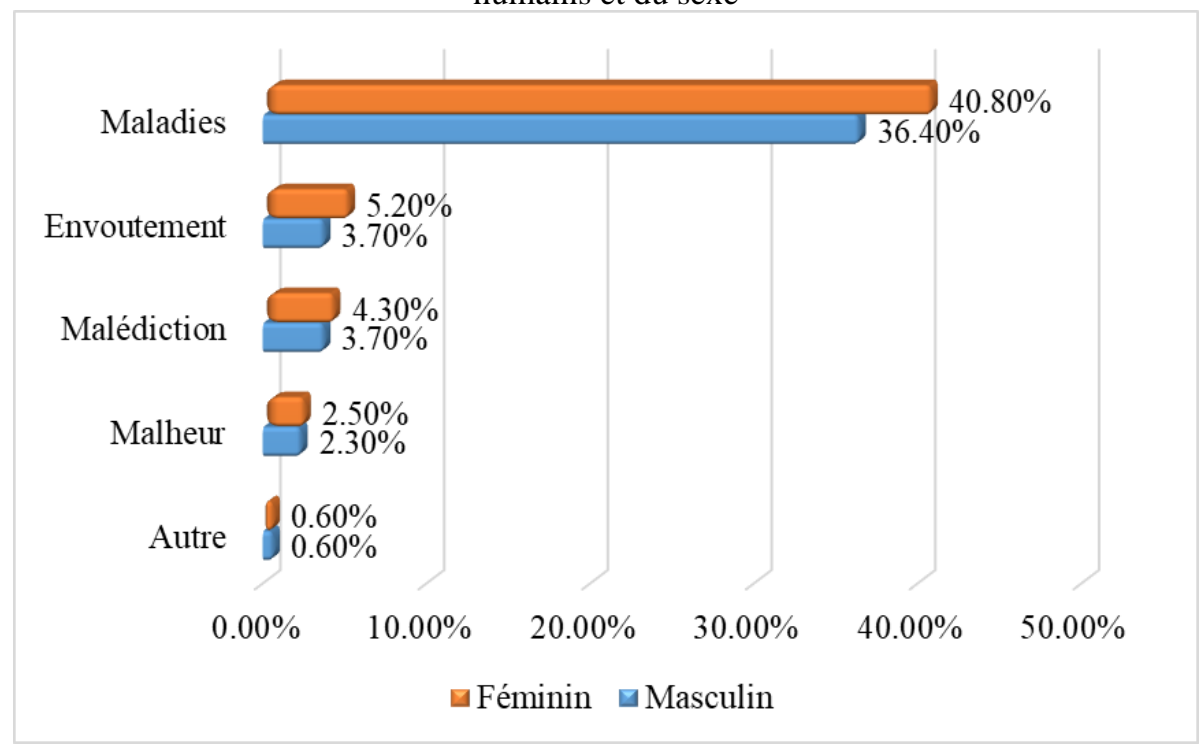

Source : Données de la recherche, juillet 2019

Il ressort de l'examen du graphique qu'indifféremment du sexe, les représentations des excréments humains mettent en exergue une idée forte à savoir la «maladie ». En effet, cette idée est soutenue à 77,2\%. Chez les femmes, elle est fortement représentée avec 40,8\%, tandis que chez les hommes elle représente $36,4 \%$ des réponses. En dépit de la domination des enquêtés de sexe féminin à avoir mentionné " maladie », quel que soit le sexe, les représentations des excréta ne changent pas fondamentalement. Cette réalité est valable autant pour les hommes que pour les femmes. Il est important de noter que le sexe n'influence pas les représentations des excréments humains. 


\subsubsection{Représentation des excréments humains en fonction de la religion}

Le graphique ci-après met en évidence les réponses des enquêtés.

Graphique $\mathbf{N}^{\circ} \mathbf{5}$ : Répartition des enquêtés en fonction de la religion et des représentations

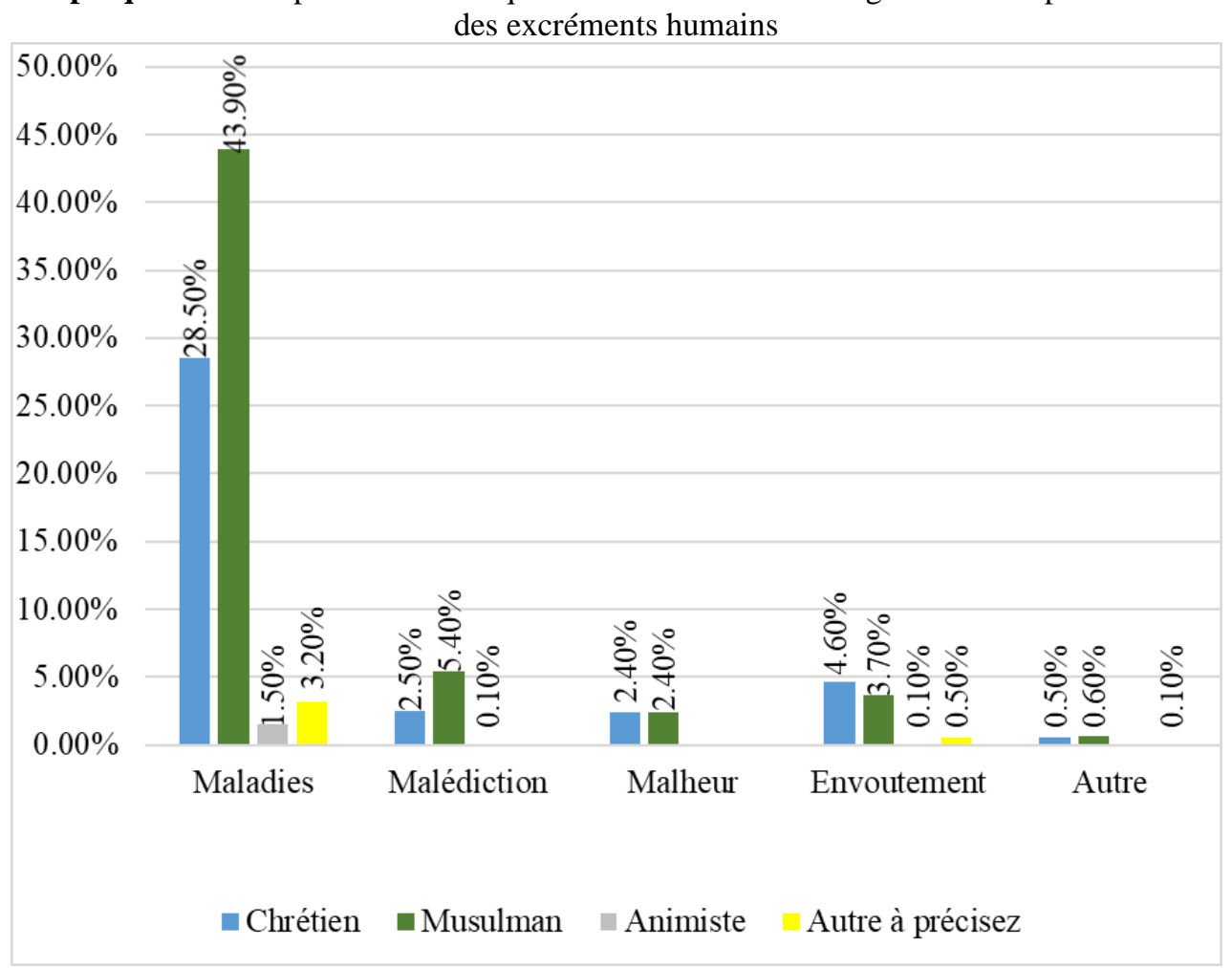

Source : Données de la recherche, juillet 2019

En suivant les données du graphique ci-dessus, concernant les représentations des excréments humains en fonction de la religion, il révèle que les enquêtés de différentes religions mentionnent plus la maladie comme étant associée aux excréments humains. Ce fait pourrait s'expliquer par les diverses formes de maladies que le contact avec les fèces occasionne par rapport aux autres maux auxquels les excréta sont associés.

Il faut noter que les enquêtés musulmans ont répondu majoritairement que la maladie et la malédiction sont associés aux fèces. Contrairement, autant de musulmans que de chrétiens font le lien entre les excréments humains et le malheur. Toutefois, il y a plus de chrétiens qui associent les excréta à l'envoutement.

\subsubsection{Représentations des excréments humains en fonction du niveau d'instruction}

Le tableau ci-dessous met en exergue le rapport entre les représentations des excréta chez les enquêtés et leur niveau d'instruction. 
Tableau $\mathbf{N}^{\circ} \mathbf{V}$ : Répartition des enquêtés selon le niveau d'instruction et de leur représentation des excréments humains

\begin{tabular}{|l|l|l|l|l|l|l|}
\hline \multicolumn{1}{|c|}{$\begin{array}{l}\text { À quoi sont associés les } \\
\text { excréments humains }\end{array}$} & Maladies & Malédiction & Malheur & Envoutement & $\begin{array}{l}\text { Autre à } \\
\text { préciser }\end{array}$ & TOTAL \\
$\begin{array}{l}\text { Niveau d'instruction } \\
\text { du répondant }\end{array}$ & & & & & & \\
\hline Non scolarisé & $36,0 \%$ & $4,3 \%$ & $2,7 \%$ & $3,5 \%$ & $0,5 \%$ & $47,0 \%$ \\
\hline Primaire & $19,8 \%$ & $1,4 \%$ & $0,6 \%$ & $2,6 \%$ & $0,4 \%$ & $24,8 \%$ \\
\hline Secondaire & $14,1 \%$ & $1,4 \%$ & $0,6 \%$ & $2,1 \%$ & $0,1 \%$ & $18,4 \%$ \\
\hline Supérieur & $7,3 \%$ & $0,8 \%$ & $0,8 \%$ & $0,7 \%$ & $0,2 \%$ & $9,9 \%$ \\
\hline TOTAL & $77,1 \%$ & $8,0 \%$ & $4,8 \%$ & $8,9 \%$ & $1,2 \%$ & $100 \%$ \\
\hline
\end{tabular}

Source : Données de la recherche, juillet 2019

Le tableau permet d'examiner les représentations des excréments humains en fonction du niveau d'instruction. Il ressort de son examen que, sur les $77,1 \%$ des enquêtés qui associent les excréments humains à la maladie, $36 \%$ sont non scolarisés et $41 \%$ ont atteint au moins le niveau primaire et au plus le troisième cycle du supérieur. Sur les $8,9 \%$ des enquêtés qui associent les excréments humains à l'envoutement et à la malédiction, $11,1 \%$ sont composés des personnes non scolarisées et ayant le niveau primaire non achevé. En ce qui concerne les réponses qui prennent en compte la malédiction et le malheur, les personnes non scolarisées sont majoritairement représentées avec $2.7 \%$.

\subsection{Croyances, tabous et langages liés aux excréments humains}

Les croyances et les tabous liés aux excréments humains forment un ensemble d'idéologies qui anime la gestion des excréta. En d'autres termes, dans les cultures africaines il existe des pensées de sens commun en rapport avec les excréta et la défécation.

\subsubsection{Croyances liées aux excréments humains}

Les excréments humains, tout comme la défécation sont animés par un ensemble d'idéologies basées sur les principes aussi bien religieux que culturels.

Sur le plan religieux, le croyant se doit d'observer certaines règles d'hygiènes. D'abord le croyant ne doit pas faire les selles n'importe où. C'est à juste titre qu'un participant affirme : "Pour le musulman, il ne peut pas s'assoir en désordre pour le faire. Il faut qu'il soit dans un endroit bien caché, il se met à l'aise, et il se nettoie propre. Un musulman ne doit pas le faire en 
désordre parce que la religion ne le permet pas. Quand tu es sale tu ne dois pas prier ». Pour le musulman tout contact avec les selles rend impur. De ce fait, le lavage anal se fait impérativement avec de l'eau, qui est considérée comme un important élément purificateur. L'eau nettoie et purifie de la saleté plus que tout autre matériel qui sert à se torcher. Autrement dit, le musulman qui est allé à la selle sans utiliser l'eau ne peut pas aller prier en portant les mêmes vêtements avec lesquels il est rentré dans les toilettes. Concernant les enfants, leurs selles ne doivent pas être déversées sur la route parce que l'enfant dont les selles ont été déversées à l'air libre pourrait tomber malade s'il advient que des passants marchent dedans.

Par ailleurs, des dispositions particulières doivent être prises par le croyant avant de faire les selles ou prendre son bain. C'est ce que explique cet enquêté quand il affirme : "En tant que des musulmans, dans l'islam, que ça soit la douche ou bien le WC c'est interdit de se positionner vers l'est, faire face au côté est quand on se lave, le soleil levant. C'est interdit dans l'islam. L'est c'est en direction de la prière. C'est-à-dire que si tu dois faire ta douche ou bien le WC, il doit être dirigé vers le sud ou le nord parce que dans l'islam, on prie tous en direction de la KAABA, de l'est, c'est sacré pour les musulmans. Il ne faut pas se soulager, se laver ou s'habiller en regardant l'est ». En effet, la douche ou bien les toilettes doivent être dirigées vers le Sud ou vers le Nord mais pas en direction de l'Est c'est-à-dire en direction du soleil levant. C'est dans cette même optique que le musulman bien averti ne se met pas face au côté Est pour prendre sa douche, faire ses besoins ou même s'habiller. L'est symbolise la direction de la prière ou de la KAABA ${ }^{1}$ qui est un lieu sacré pour les musulmans. Mais, les habitations ne respectent pas ces préceptes. Les douches comme les latrines sont construites sans tenir compte de cette réalité. Aussi, devant les difficultés d'accès aux logements à coût abordable, pour la plupart des ménages locataires, la première des préoccupations c'est de pouvoir se loger en dépit des principes religieux.

Dans le contexte culturel ivoirien, le fait d'aller déféquer devant ou dans une maison, un lieu de commerce ou un champ, signifie que celui qui agit ainsi pose un acte de sorcellerie dirigé envers celui à qui appartient la maison, pour détruire la personne qui y vit ou porter atteinte à l'activité de celui-ci. Les propos de ce enquêtés traduisent bien ce fait : "On va en brousse c'est vrai mais tu vas pas aller dans un lieu sacré pour aller faire ça. Dans le champ on peut le faire, dans la forêt. Mais tu vas pas aller faire ça dans le champ de ton voisin, il va voir ça il va mettre médicament dessus. C'est ta mort tu cherches. Tu peux trouver un endroit sécurisé dans ton propre secteur pour pourvoir faire ça». Par conséquent, ce dernier peut utiliser les fèces de celui qui a commis le méfait pour renvoyer le sort. En d'autres mots, sur le plan

${ }^{1}$ Lieu saint de l'Islam vers lequel se tournent les croyants pour prier. 
surnaturel, les excréments d'une personne peuvent être utilisés contre sa personne. Aussi, selon certaines conceptions, les endroits comme la douche et les toilettes sont fréquentés par les génies. De ce fait, il n'est pas conseillé de communiquer ou même de parler une fois dans ces endroits, cela donnerait l'occasion aux génies de découvrir ta nudité et même de te rendre malade. Le fait de parler peu attirer sur toi le regard des génies.

Tout ceci constitue un ensemble d'idéologies développer par rapport aux excréments humains. Le fait d'aller à l'encontre de ces prescriptions peut provoquer un évènement malheureux. Pour quelqu'un qui connait le sens de ces choses et qui le fait, peut avoir un malheur. Il en est de même pour celui qui pose ces actes sans pour autant connaitre les implications.

\subsubsection{Tabous liés aux pratiques de la défécation}

Il est question ici des pratiques d'évacuation des excréments humains considérées comme défendus dans nos sociétés africaines. Il existe des interdits liés aux lieux de défécation. Cette affirmation d'un chef de communauté semble confirmer cette réalité : "Il ne faut pas s'assoir en désordre pour le faire. Il faut que ça soit dans un endroit bien caché ». Les propos de cet autre participant confortent ceux de son prédécesseur lorsqu'il affirme : "Que tu sois musulman, chrétien, de n'importe quelle ethnie, tu dois savoir qu'il y a certains coins même si on ne vit pas dedans, on ne doit pas faire la saleté dedans ». Ces affirmations, donnent à entendre qu'il y a des lieux indiqués et des lieux non indiqués pour faire les selles.

Cependant, l'espace peut être extérieur à l'homme mais habité par les êtres mystiques qui président certains lieux et de ce fait sont interdits à la défécation. Par exemple, les lieux sacrés sont des endroits qui ne sont pas propices parce qu'ils sont habités par les génies. De même, il est interdit de déféquer dans les champs étant donné que ce qui est cultivé est destiné à la consommation. De plus, le champ est considéré comme le lieu de travail des personnes qui cultivent la terre. Ainsi, aller déféquer dans le champ d'une personne est considéré comme une provocation. Aussi, en bordure de la mer ou de la lagune, où il y a des habitations, il est interdit de déféquer à proximité des maisons. Il en est de même pour les routes principales, les lieux où la population se réunie, tous les endroits qui sont fréquentés, comme les alentours des arbres fruitiers.

Déféquer dans un de ces endroits peut être dangereux pour la personne. Ceux qui fréquentent ces lieux auront à dire des paroles qui peuvent atteindre la personne qui a commis l'acte. La personne qui va déféquer dans ces lieux, attire sur lui la malédiction. Il peut par conséquent être frappé d'une maladie ou d'un mal d'origine inconnue. Ces déclarations d'un chef de communauté étayent ce fait: "Les animaux boivent l'eau de la rivière, quand il n'y a pas l'eau, nous-même on se ravitaille là-bas, quand tu chies dans une eau qui ne 
coule pas, ça attire la malédiction. L'eau qui coule ça c'est acceptable, on sait que ça peut avancer. Même si caca te serre il y a des distances, il faut te déplacer. Sois-tu creuses un trou, tu chies et tu fermes. Le plus souvent chez nous c'est comme ça. On creuse un trou quand on a fini de chier on ferme, on appelle ça soutra, mé ka bokè soutra là, i ka datoukou soutra là. Quand on dit soutra c'est-à-dire tu vas au besoin dans un endroit où on n'entend pas les bruits que tu fais, parce qu'il y a certaines personnes quand ils vont chier, ils font beaucoup de bruits prouprou, c'est une humiliation ». À l'analyse de ce qui précède, il ne faut pas laisser ces selles à l'air libre à la vue des autres, ou bien déféquer dans les lieux qui servent à tout le monde. Autrement dit, l'acte de défécation doit se faire en toute discrétion parce qu'il suscite la gêne, l'opprobre. Il faut souligner que une fois en ville, nombreux sont ceux qui laissent derrière eux les pratiques culturelles parce que le nouvel environnement social ne s'y prête pas. De ce qui précède, il ressort que, d'un lieu à l'autre, d'une société à une autre les pratiques diffèrent, de ce fait, les déplacements exigent de s'adapter au nouvel environnement.

\subsubsection{Désignation des excréments humains dans les langues locales}

Les excréments humains dans les langues locales ont une signification qui permet de comprendre les représentations sociales qui y sont rattachées. Les questions suivantes ont permis de saisir ce lien :

Comment dit-on déchets humains dans votre groupe sociolingustique? À quoi d'autre ce mot est-il associé ?

Selon les Godiés ${ }^{2}$, il y a un lieu où tout le monde vient faire ces besoins. En témoigne, les propos de ce enquêté : "Chez nous n'y a pas WC on s'arrête sur un bois et puis on chie, nous on ne chie pas dans maison, puisque on est arrivé il y a maison ici, on se lave et puis on chie en même temps. Gbégbé là c'est la clôture ou on se lave. Maintenant, si ici on se lave et puis on chie en même temps, chez nous on dit gnrigbégbé, donc dans gbégbé là c'est là on chie, c'est-à-dire que c'est maison de caca. Ce n'est pas avec lotus, c'est avec bambous ou bien bois ou bien feuille. Tu vas prendre bambou pour t'essuyer, c'est comme ça. Mais comme on est à Abidjan ici c'est là il y a WC, il y a lotus on prend pour se nettoyer $»$. De cette assertion, il ressort que chez les godiés, les lieux de défécation ont différentes appellations. Il y a une distinction entre le lieu où on va déféquer et les latrines. Le premier peut être un endroit indiqué pour faire les selles. En langue local godié, il est désigné par: « gnrignondan », «Gnri » ce sont les excréments humains, «Gnon » signifie tu chie, «dan » c'est le lieu. Littéralement, « gnrignondan » désigne "le lieu où tu vas déféquer". Par contre, le deuxième indique les latrines qui sont en

${ }^{2}$ Peuple qui vient de Fresco et de Sassandra, villes situées sur la côtière dans le sud-ouest de la Côte d'Ivoire. 
fait une pièce (quatre murs) où se trouve une installation sanitaire servant à recueillir ou à évacuer les excréta. En godié, les latrines sont appelées " gnrigbégbé ». Le mot est composé de «gnri » qui signifie excréments et de " gbégbé » qui désigne la maison. C'est la maison où l'on va faire ses besoins donc la «maison de caca». Une fois en ville, les habitudes changent. La défécation se fait dans un endroit clos ou une maison. Par ailleurs, une autre distinction est observée pour ce qui est du matériel utilisé pour la toilette anale. Tandis qu'en milieu rural les moyens de bord sont utilisés (bois, bambous, feuilles), l'avènement des toilettes impose un matériel requis, l'usage du papier hygiénique.

Cette représentation des lieux de défécation est aussi observée chez les attiés. À cet effet, un participant affirme : "Excrément c'est sun en Attié. Quand tu veux aller au WC, l'endroit où on va déféquer s'appelle sunkoi. Puisque que on est tous venu à Abidjan et puis y a un lieu où on doit se laver et puis il y a le WC en même temps le lieu où on se lave là s'appelle pkassun. Il y a le coin où on va au WC qui est sun, où on se lave qui est pka. Le lieu où on va au WC et on se lave en même temps qui est pkassun c'est à dire tu te laves et maintenant tu vas au WC. Au village où il n'y pas de WC le lieu où on va déféquer se dit sunkoi. » Il est important de noter que très souvent, le mot qui désigne les excréments est utilisé pour désigner aussi l'endroit qui sert de lieu de défécation ou est associé à un autre mot.

En dida ${ }^{3}$ par contre, «gnribété » indique à la fois la prison et l'endroit pour faire les besoins parce que comme l'explique cet enquêté au sujet de la prison, «c'est le lieu où on chie et puis on dort là-bas en même temps ». Cette observation est faite aussi en baoulé ${ }^{4}$. Le mot «bisoua» composé du mot «bi»qui signifie caca et de «soua» qui veut dire maison, traduit littéralement la maison de caca qui désigne en fait la prison. Dans les langues locales ivoiriennes comme dans les langues étrangères rencontrées, les excréments humains sont toujours associés aux déchets ou à la saleté. Ainsi, les dioulas, maliens, malinké utilisent le mot "gnaman» ou "gnanmangnanman» pour désigner à la fois les excréments humains et la saleté. De ce fait, il serait mal vu de prononcer ou de dire directement que tu vas à la selle selon que tu es dans tel ou tel autre endroit. En effet, cela peut même s'avérer insultant pour ceux qui sont en face de toi. Comme le fait remarquer en substance cet enquêté qui affirme : "Quand tu respectes les gens qui sont en face de toi, il y a la manière que tu adoptes quand tu vas aux toilettes. Tu dois respecter ceux qui sont autour de toi, ils ne doivent pas savoir ce que tu vas faire. Et la manière dont tu te nettoies. Il y a une main qu'il faut

\footnotetext{
${ }^{3}$ Langue locale parlée par les populations du centre-sud de la Côte d'Ivoire précisément dans la ville de Lakota, Divo

${ }^{4}$ Langue local ivoirienne parlée par le peuple du centre de la Côte d'Ivoire notamment dans les villes de Bouaké, Yamoussoukro...
} 
utiliser, il y a une main que tu ne dois pas utiliser ». Cette autre assertion d'un habitant rend compte de cette réalité : "Il y a des attitudes à ne pas avoir dans certains endroits où tu te trouves. Ici par exemple il y a certains qui n'ont pas de WC, si quelqu'un qui n'a pas de WC ditje vais me mettre à l'aise, c'est que soit il va dans une douche publique où il va s'assoir en désordre pour le faire. »

En malinké 5 il faut plutôt utiliser l'expression « en mé ta djassa la » ce qui veux dire je vais dans les ordures, les selles étant considérées comme des ordures. Les grandes personnes savent ce que cela signifie mais les enfants ne le savent pas. Dire directement «mé ta cabiné la » qui veut dire : "je vais au $W C^{\prime \prime}$ devant une grande personne, est une expression vulgaire. De plus les personnes qui n'ont pas la maitrise de la langue utilisent le mot cabinet emprunté au français pour traduire qu'ils vont à la selle. Les attiés ${ }^{6}$ disent plutôt «je vais derrière le mort» "mi kan zé » pour dire "je vais déféquer" ou «mè zénikan » je vais au besoin au lieu de «Mé zeu sun » pour dire "je vais déféquer" qui est trop grossier. Celui qui respect les autres dit «mi kan zé ». En denguélé les excréments humains ce disent «bô » ainsi, dire directement «mé ta bô kè » ne suscite pas le respect pour ton entourage. Il faut plutôt dire «mé ta sokoman » "je vais derrière la maison" parce qu'au village c'est derrière la maison que se trouve le lieu de défécation. Une autre manière d'aller à la selle avec pudeur est de partir sans dire mot à qui que ce soit, dans ce cas personne ne sait ce que tu es censé faire.

C'est avec la colonisation que les WC ont été introduits en Afrique. Auparavant, il y avait des endroits qui faisaient office de lieu de défécation comme les dépotoirs, la broussaille loin des voies empruntées, des cours d'eau, des plantations. Ce fait existe toujours dans certaines localités. Les pratiques de défécation sont intégrées dans les coutumes de chaque peuple. Très souvent, les populations se déplacent avec leur culture. Il n'est donc pas étonnant de voir que ces pratiques soient transposées en ville.

\section{Discussion}

Les préoccupations d'ordre environnemental en général, la question de la gestion des excréments humains en particulier révèlent des enjeux à la fois environnementaux, politiques, économiques et sanitaires. Pour comprendre les pratiques en matière de gestion des excréta, il faut saisir le sens que les populations accordent aux excréments humains. La théorie des représentations sociales mobilisée à cet effet est définie comme "une forme de connaissance socialement élaborée et partagée ayant une visée pratique et concourant à la construction d'une réalité commune à un ensemble social » (Jodelet, 1984, p.

\footnotetext{
${ }^{5}$ Peuple du nord-ouest de la Côte d'Ivoire principalement dans les villes d'Odienné, de Kong ${ }^{6}$ Peuple du sud-est de la Côte d'Ivoire. Dans la ville d'Abidjan on les retrouve de la commune d'Anyama
} 
36). Pour Pierre Mannoni (1998), lorsqu'on traite les représentations sociales, l'un des principaux problèmes qui se pose est de savoir quelles sont leurs limites, leurs contours mais aussi l'étendu du champ social concerné, les référents culturels évoqués explicitement ou implicitement, les mécanismes intrapsychiques conscients ou inconscients impliqués, les pratiques sociales ou les processus psychologiques à l'œuvre, les cadres institutionnels ou simplement sociaux intéressés. En somme, les représentations régissent les pratiques sociales mais également influencent les attitudes par le " jeu représentationnel ». Les représentations sociales construites autour des excréments humains se perçoivent à travers "un ensemble de connaissances, d'attitudes, de croyances » (Flament, et Rouquette, 2003, p. 13).

Dans la plupart des langues locales rencontrées (Baoulé, attié, malinké, Godié, Dida), les excréments humains désignent en général les déchets ou la saleté. Les différentes appellations des excréments humains n'ont qu'une seule signification. Elles traduisent quelque chose qui suscite le dégout, l'éloignement voire le rejet. À cet effet, Berger et al (2010) dans leur étude font remarquer que les soignants éprouvent des « sentiments dévalorisant et honteux » face à la prise en charge de l'élimination intestinale. Parler d'élimination intestinale suscite de la gêne autant pour le patient que pour le personnel de santé parce que c'est un sujet d'intimité « ..., caractérisée par la saleté et les mauvaises odeurs » (Hoerni, 2000 cité par Berger et al 2010, p. 72). Dans cette même optique, N'Goran révèle à travers son étude que les Odzukru et les Senoufo développent des réactions de rejet à l'égard des excréta. Pour eux, les fèces à l'état pur constituent une matière dégoutante et répugnante. Les excréments humains ont une image péjorative. De ce fait, en parler requiert de prendre des dispositions comme voiler par des mots imagés le fait d'aller déféquer ou éviter d'évoquer le sujet dans certains endroits. N'Donko qui va dans le même sens soutient que: "La société, quelle qu'elle soit, fixe des normes afin de contrôler la manière de faire et de dire, en matière de déchets du corps » (N'Donko, $1988: 13$ ). Certaines expressions "d'après le dire des Yasa, sont crues et offensent la pudeur. Elles ne sont utilisées d'autant qu'il soit commode d'en parler qu'entre personnes d'une même classe d'âge» (Ibid : 48). Parler des excréments humains nécessite d'adopter des manières délicates dans le langage.

Par ailleurs, dans certaines langues locales comme en Baoulé par exemple, le mot excrément est associé à la prison en raison du fait que dans le lieu qui servait autrefois à détenir les prisonniers, ceux-ci faisaient en même temps leurs besoins. Le Breton (2003) va encore plus loin sur les questions liées aux représentations et à la défécation en tant que pratique humaine. Pour lui les notions de honte, d'intimité, de pudeur, de sensibilité ce sont développées au fur et mesure de l'évolution sociale et de l'organisation familiale. À telle point que pour assouvir certains besoins naturels comme la 
défécation, des endroits sont réservés à cet effet, assurant ainsi l'intimité de chacun. L'anthropologue David Le Breton explique que la défécation est devenue sujet de honte impliquant le silence, le repli sur soi et surtout la crainte d'être surpris en si fâcheuse posture. Pour Martin et al (2004) «L'embarras à parler franchement des excréments humains constitue un obstacle à l'assainissement de l'eau à l'échelle mondiale alors que près de la moitié de l'humanité n'a pas droit à des toilettes » (Martin et al, 2004, p.7). De plus, audelà de la gêne ou de la honte que peut susciter le sujet, Douglas (1971) observe que le contact avec les excréments inspire une attitude de dégoût. À tel point que dans la société Hindou, ce sont les personnes de castes inférieures qui nettoient les toilettes. Dans son ouvrage « De la Souillure », il affirme que les excréments sont des agents de pollution rituelle et que le nettoyage des latrines est réservé aux castes inférieures. Plus loin, il explique que les déchets corporels sont symboles de danger et de pouvoir.

La défécation est un besoin naturel et indispensable pour tout être humain. Cet acte, bien qu'individuel, implique la société toute entière qui a mis en place toute une organisation pour arriver à une bonne gestion des excréments humains. À cet effet, les sociétés humaines ont leurs systèmes de représentation sociales sur lesquels se fondent la gestion des excréments humains. La défécation est une activité physiologique commune à tous les hommes, quel que soit leur condition, leur culture. Cet acte banal qui se veut très intime s'inscrit pourtant dans toute une réglementation sociale (N'Donko, 1988, p. 23). Les résultats de notre étude montrent effectivement qu'il existe un ensemble de règles qui régissent la gestion des excréments humains. Il se résume en des interdits. Ainsi, il est interdit de déféquer dans certains endroits comme les rivières, les champs, derrière l'habitation d'autrui. Sur ce point, N'Donko (1988), aborde dans le même sens. Selon l'auteur, le bord de la mer est le lieu de défécation par excellence chez les Yasa. Elle est un grand purificateur qui efface toute la saleté déposée à la plage. D'autres zones de défécation potentielles sont la brousse et la rivière. Mais, aller en forêt ou ailleurs n'est pas socialement acceptable et constitue une déviation par rapport aux normes admises. Par ailleurs, les Yasa construisent des latrines pour ne pas être pénalisé. Ainsi, " avoir des latrines est une chose, les utiliser en est une autre ». Ils n'utilisent les latrines que pour les cas de maladie par exemple ou comme palliatif nocturne.

Par ailleurs, les conséquences peuvent être lourdes pour les personnes qui ne respectent pas les règles établies en matière de défécation. En fait, ceuxci peuvent être du fait des plaintes et des paroles proférées contre eux, sujet de malédiction ou même contracter des maladies d'origines inconnues. Les excréments humains peuvent être à l'origine de divers maux. Ainsi, l'individu peut être envouté, maudit à travers ses selles. Ces résultats corroborent avec ceux de N'Donko (1988), dans laquelle il démontre que les excréments 
peuvent être utilisé dans le cadre de "magie maléfique » contre des personnes pour les enchanter, envouter ou les rendre malade. Ainsi, «les matières fécales entretiennent des liens fort sympathiques avec l'individu» (op cit : 54). De même, N'Goran (2016) révèle que pour les peuples Odzukru et les Senoufo, les fèces portent en chaque individu, des traces de sa vie qui peuvent éventuellement être utilisé contre sa personne. Notre étude a montré que dans l'ensemble les excréments humains sont considérés comme objet de mal. De ce fait, ils peuvent être utilisés pour mettre fin à la vie. Raison de plus pour s'en éloigner. Le regard que porte les individus sur les excréments, comment ils les évacuent, varient d'une culture à l'autre.

\section{Conclusion}

À travers ce sujet «Pratiques liées aux représentations sociales des excréments humains chez les populations des quartiers défavorisés du District d'Abidjan », il s'agit, de répondre à la préoccupation suivante : quelles sont les représentations sociales des excréments humains chez les populations des quartiers défavorisés ? La démarche méthodologique mise en place à cet effet a mobilisé des outils et techniques d'une approche mixte. Ce sont: le questionnaire, le guide d'entretien, l'observation et l'échantillonnage. Pour l'analyse des données, le logiciel utilisé est sphinx ${ }^{2}$ V5 pour les données quantitatives et la méthode d'analyse de contenu thématique pour les données qualitatives.

Au terme de notre recherche, il faut noter que les définitions des excréments humains se rapportent essentiellement à la saleté. Les définitions des enquêtés ne diffèrent pas en fonction du sexe, de la religion et du niveau d'instruction. Les excréments humains sont perçus comme synonyme de mal en ce sens que les fèces peuvent être cause de maladie, d'envoutement, de malédiction ou de malheur. De ce fait, ils sont perçus comme tel par les populations indépendamment de leur sexe, de leur niveau d'instruction et de leur religion. Les résultats de cette étude montrent aussi que les langues locales rendent compte des représentations sociales des excréments humains.

En somme retenons que la façon dont les populations disposent de leurs excréments est une question très personnelle, tellement intime d'ailleurs que dans la plupart des cultures on n'aborde pas franchement le sujet. Il y a beaucoup de gens qui pensent que le mot « excrément» est un gros mot, ou un mot « sale », surtout s'il est utilisé en présence d'autres personnes. De plus, on n'aborde pas ouvertement le sujet dans les questions d'assainissement et d'hygiène parce que beaucoup de personnes trouvent le sujet gênant.

\section{References:}

1. BOURKE, J.-G., (1981). Les rites sociologiques, philosophie d'aujourd'hui, Paris : PUF. 
2. BERGER, V. ; DURAND, L. ; GROCQ, Martine., (2010). Élimination intestinale à l'hôpital réflexion éthique sur sa prise en charge par les soignants in Recherche en soins infirmiers $\mathrm{N}^{\circ} 103$-DÉCEMBRE-pp 67-77 [en ligne]https://www.cairn.info/revue-recherche-en-soinsinfirmiers-2010-4-page-67.htm\#

3. ELIAS, N., (1976). La civilisation des mours, $1^{\circ}$ édition 1939, $2^{\circ}$ édition 1696, Collection Agora, Calmann-Levy, Pocket, 342 pages.

4. FLAMENT, C. ; ROUQUETTE, M.-L., (2003). « Anatomie des idées ordinaires »Comment étudier les représentations sociales, Armand Colin, 176 pages.

5. Institut National de la Statistique (INS), 2016. Multiple Indicator Cluster Survey.

6. JODELET, D., (1984). Représentation sociale : phénomène, concept et théorie, Paris PUF, pp. 357-378.

7. KOUADIO, A.R.; TRA, F.; OUATTARA, S., (2021). Représentations sociales de la pollution de l'air intérieur et pratiques sociales des malades d'asthme de la commune de Yopougon (Côte d'Ivoire). European Scientific Journal, ESJ, 17(14), 217. https://doi.org/10.19044/esj.2021.v17n14p217

8. LAWLER, J., (2002). La face cachée des soins, Ed Seli Arslan, p 71. 288 pages.

9. LE BRETON, D., (1997). De l'excréta en anthropologie, Voyage sans transit, Éditions Medigone pour les laboratoires Beaufour, Paris, pp 815.

10. Le Grand Larousse illustré (2017).

11. MANNONI, P., (1998). Les représentations sociales. PUF, 127 pages.

12. MARTIN, P.; LIENARD, C. ; MARIN, C. ; ADAM, X. ; et GANDIN, E., (2004). www.amisdelaterre.be. Perspectives d'évaluation : L'eau et l'assainissement en milieu rural, Numéro 6 . Juillet 2012, 8 pages.

13. Ministère du plan et du développement, la situation des femmes et des enfants en Côte d'Ivoire Enquête par grappes à indicateurs multiples - Côte d'Ivoire 2016 MICS5. 442 pages.

14. N'DONKO, F., (1988). Représentations et pratiques culturelles liées aux excréments humains et autres déchets du corps chez les populations de la savane et de la forêt (côtière). Dissertation doctorale pour l'obtention du DEA en anthropologie, 109 pages.

15. N'GORAN, S.I., (2016). Déterminants socio anthropologiques de l'utilisation des excréta humains hygiénisés comme engrais en milieu rural ivoirien : cas des paysans Odzukru et Senoufo, Thèse de Sociologie Université Félix HOUPHOUËT-BOIGNY, 324 pages. 
16. N'GUESSAN, M., (2016). Les représentations de la santé et de la maladie chez les Agni Sanwi. Thèse unique de Doctorat de Sociologie, Université Félix Houphouët Boigny. 341 pages.

17. Organisation des Nations Unies pour l'Education, la Science et la Culture (UNICEF) (2008). International Year of Sanitation. En ligne, www.sanitationyear2008.org consulté le samedi 12 août 2017, $22: 22$ $: 46$.

18. PNUD. (2013). «Etudes des quartiers précaires dans les treize (13) communes du District d'Abidjan et définition des plans de restructuration

http://www.ci.undp.org/content/cote_divoire/fr/home/presscenter/arti cles/2013/11/28/20-des-habitants-du-district-d-abidjan-vivent-dansles-quartiers-pr-caires.html consulté le 26/09/2017 à 17h50. 